February 1988

\title{
PROPOSAL TO THE DEPARTMENT OF ENERGY FOR PARTICIPATION IN THE UA1 EXPERIMENT
}

\author{
James W. Rohlf \\ Associate Professor of Physics \\ High Energy Physics Laboratory \\ Harvard University \\ Cambridge, MA 02138
}

\begin{abstract}
This proposal is to the Department of Energy for 501.6K dollars (349.6K operations and $152 \mathrm{~K}$ equipment) for continued participation in the UAl experiment on proton-antiproton collisions.
\end{abstract}

\section{DISCLAIMER}

This report was prepared as an account of work sponsored by an agency of the United States Government. Neither the United States Government nor any agency thereof, nor any of their employees, makes any warranty, express or implied, or assumes any legal liability or responsibility for the accuracy, completeness, or usefulness of any information, apparatus, product, or process disclosed, or represents that its use would not infringe privately owned rights. Reference he ein to any specific commercial product, process, or service by trade name, trademark, manufacturer, or otherwise does not necessarily constitute or imply its endorsement, recommendation, or favoring by the United States Government or any agency thereof. The vicws and opinions of authors expressed herein do not necessarily state or reflect those of the United States Government or any agency thereof. 
CONTENTS:

I. Introduction 3

II. Data Analysis 4

III. Hardware: Position Detector 5

a) Need for the Position Detector

b) Mechanics

c) Electronics

d) Results of Beam Tests

e) Time Schedule and Costs

IV. Budget 26

References $\quad 28$

Appendix $\quad 29$ 


\section{Introduction}

The UAl experiment is the study of high-energy proton-antiproton collisions in the Super-Proton-Synchrotron (SPS) Collider at CERN. A major upgrade of the UAl detector is in progress for operation with the upgraded antiproton source (ACOL). The U.S. groups have played an increasingly prominent role in UA1 during the past few years. The U.S. team consists of groups from Harvard, MIT and UCLA (with the UC Riverside group moving to the DO experiment at Fermilab). A joint U.S. teain equipment request for UA1 was made in March 1987 [1]. The Harvard physicists participating in UA1 currently include G. Bauer, S. Geer, G. Pancheri (part-time), J. Rohlf, C. Rubbia, K. Sumorok, and three graduate students, Mr. C. Jessup, Mr. J. Kroll, and Mr. A. Schwartz. The operations of this group have been covered by the Rubbia task[2] of the Harvard High Energy Physics Contract with the Department of Energy. In addition, J. Rohlf has been supported, in part, by the DOE Junior Investigator Program[3]. Next year will see a major change in the Harvard DOE contract[4] with Professor Carlo Rubbia leaving Harvard to become Director General of CERN. 


\section{Data Analysis}

The Harvard group has been strong on many aspects of data analysis since the beginning of UA1. The most significant UA1 results obtained to date are: 1) the observation of the $W$ and $Z$ intermediate vector bosons and measurement of thiir basic properties, providing the ultimate confirmation of the $\mathrm{SU}(2) \times \mathrm{U}(1)$ unified theory of electromagnetic and weak interactions, and 2) measurement of properties of hadronic jets arising from parton (quark and gluon) scattering, thereby directly verifying both qualitatively and quantitatively the validity of the quantum-chromodynamic $(Q C D)$ theory of strong interactions.

We have established a data processing and analysis center for UA1 at the Harvard High Energy Physics Laboratory in collaboration with MIT. The core of this is a 4361 mainframe computer purchased with a substantial discount by special arrangement between Harvard University and IBM Corporation. This computer is used to read and write data through a specially developed VME interface connected to two homemade 3081 emulators which each have the CPU power of approximately a VAX 8600 . We have planned to expand the facility to ten emulators. Details of the system configuration and performance may be found in ref. [1]. We also have two Megatek work stations connected to a VAX 8600 , which are used for UA1 analysis.

The UA1 experiment is expected to receive a total integrated luminosity of more than 30 $\mathrm{pb}^{-1}$, more than a factor of forty beyond the present data sample. We expect to process a significant fraction (about one-third) of this data in Cambridge. We plan to put emphasis on 1) more precise measurement of $\mathrm{W}$ and $\mathrm{Z}$ properties, 2) tests of $\mathrm{QCD}$ to higher order in the strong coupling constant $\alpha_{s}, 3$ ) searches for new quarks and leptons, 4) search for the Higgs, and 5) search for unexpected and unproved phenomena (such as supersummetry). We feel that our experience with high-energy hadron collisions together with our upgraded second-generation detector running at the high luminosity $\mathrm{ACOL}$ will enable us to complement and compete well with other existing physics programs over the next few years. 


\section{Hardware: Position Detector}

Hardware responsibilities have included the routine running and maintenance of the UA1 detector at CERN. We have been active in the design and construction of the uranium-tetramethylpentane (TMP) calorimeter which constitutes the heart of the UA1 hardware ungrade[4]. For this, we have taken special responsibility for the specification and fabrication of 300 tons of uranium plates. We also developed with Wylie Organic a procedure to produce a large quantity of highly purified TMP. We were responsible for a Fastbus system of TDC's for readout of a micro-vertex detector. The major inovative component was a special Fastbus-VME interface designed and built at Harvard enabling readout of the Fastbus memory at $10 \mathrm{MHz}$. The micro-vertex detector will not be used for the ACOL running, but the electronics we built worked well and will be used by another experiment at CERN.

The major hardware activity currently is the construction of the TMP position detectors for the upgraded UA1 calorimeter. The progress of this is decribed in detail in the following sections.

\section{a) Need for a Position Detector}

The upgraded UA1 detector which will run at the CERN SPS Collider with the new Antiproton Collector Ring (ACOL) is a second-generation device designed for precision calorimetric measurements. The position detector will significantly enhance the physics capabilities of this new detector. The main functions of the position detector will be 1) the precision position measurement of electrons from $\mathrm{W}$ and $\mathrm{Z}$ decays which are required for accurate mass measurements, 2) the identification of isolated electrons in order to search for the semi-leptonic decays of new objects (such as the top quark), and 3) the identificition of single isolated photons.

The position detector will also be used to check and monitor the absolute calibration of the TMP calorimeter. The position detector will allow the reconstruction of $\pi^{0} \rightarrow \gamma \gamma$ decays by measurement of the energies and opening angle of the two photons, providing a check aganst the known $\pi^{0}$ mass. 


\section{b) Mechanics}

The position detector is a TMP liquid ionization chamber with a triple electrode construction. The center electrode has a pad structure like the other calorimeter boxes. The other two electrodes have an orthogonal strip configuration. The position detector is the seventh box in the TMP-uranium stack, being placed after about four radiation lengths of absorber in order to collect a large charge over a wide range of electron or photon shower energies. The position detector is compatible with the calorimeter design for many practical points such as mechanical placement, electronic readout, radiation resistance, high voltage distribution, hermiticity and energy resolution.

The position detector is being constructed under our supervision at Hutchinson Technology. The position detector contains 24 horizontal strips, 56 vertical strips and 8 center electrodes. A photograph is shown in fig. 1. The electrodes have a width of $7 \mathrm{~mm}$ and a thickness of $0.5 \mathrm{~mm}$. The space between electrodes is $2.1 \mathrm{~mm}$. The thickness of the stainless steel covers is $0.150 \mathrm{~mm}$. The electrodes are held apart by ceramic spacers and the high voltage is connected to a specially designed feedthrough. The boxes are sealed to a leak rate of $10^{-9}$ torr-liter/s with high technology laser welding. The assembly is done in a clean room with special attention to the handling of all components during production.

Twelve full-sized units have been completed at Hutchinson. Materials are being acquired for the gondola production ( 350 units). A total of 480 position detectors are needed for the entire TMP calorimeter.

The TMP filling and stack assembly is done at CERN. 


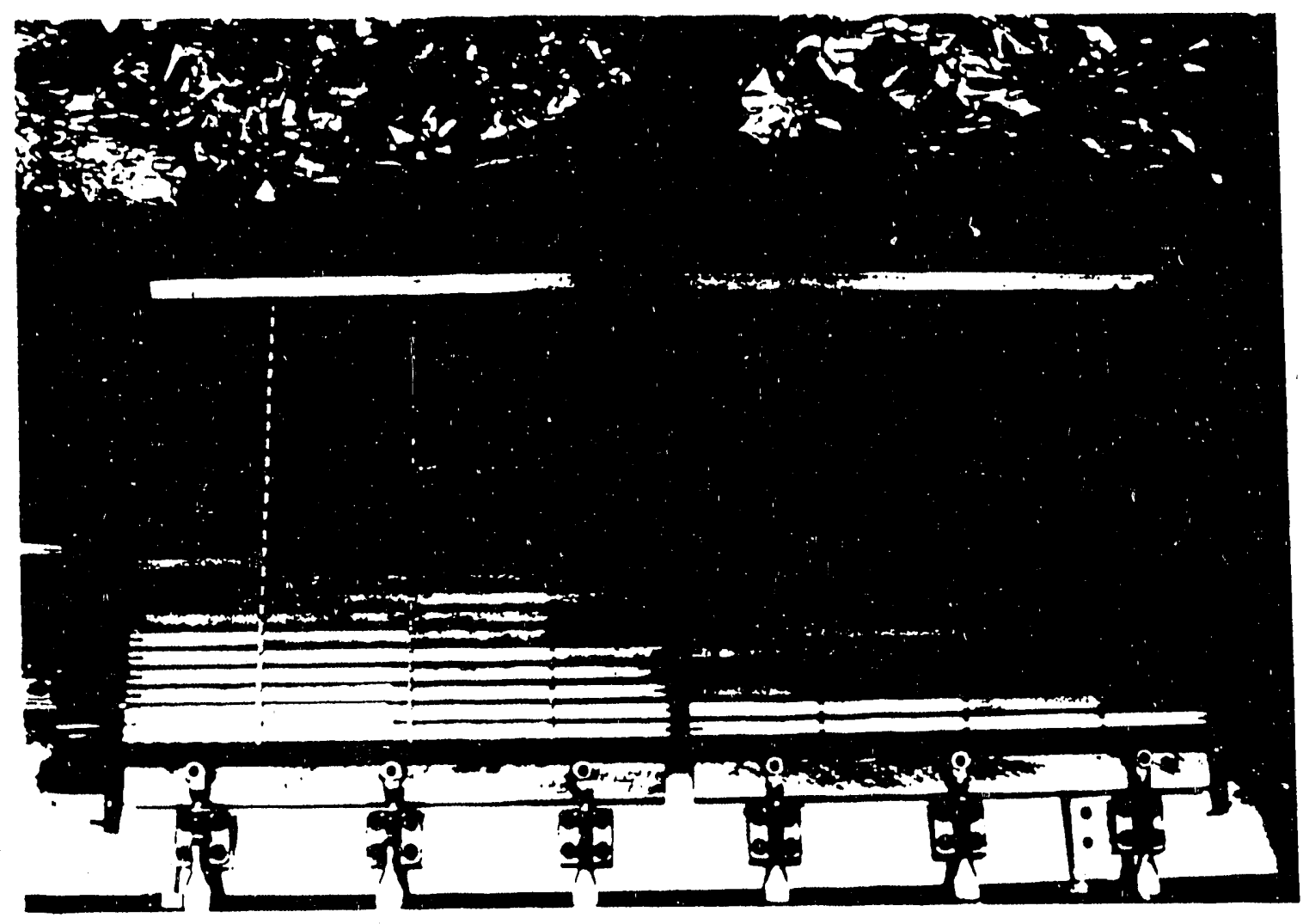

Figure III.1 a) Photograph of full-sized position detector module built at Hutchinson Technology, showing horizontal strips. 
Figure III.1 b) Photograph of full-sized position detector module built at Hutchinson Technology, showing horizontal strips and center electrodes.

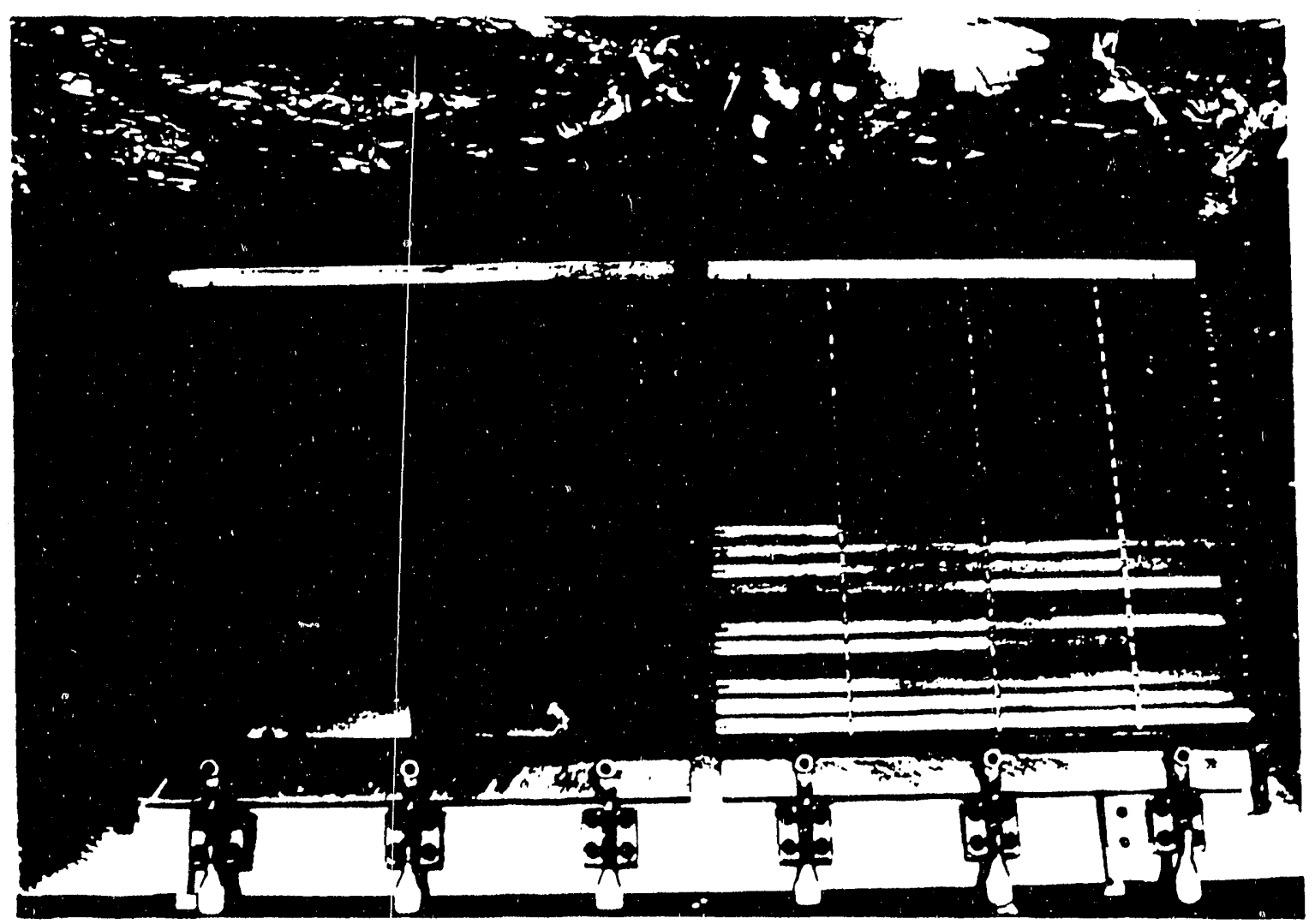




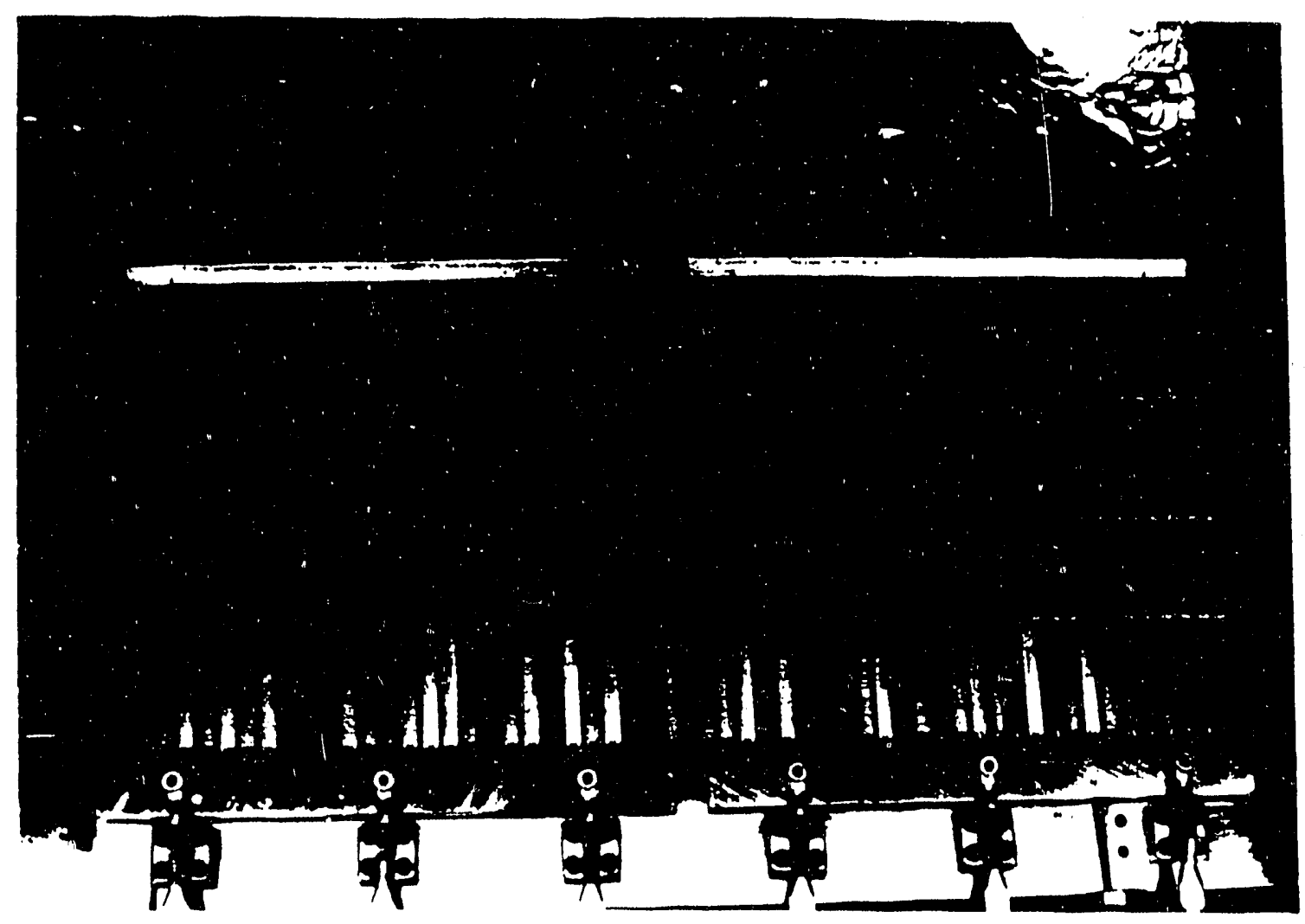

Figure III.1 c) Photograph of full-sized position detector module built at Hutchinson Technology, showing vertical strips. 


\section{c) Electronics}

The position detector has $37.4 \mathrm{~K}$ active elements. The main components of the position detector electronics are preamplifier hybrids, calorimeter data units, preamplifier motherboards, $A D C$ 's, time sequencer, memory address sequencer, and flex receiver. The status of the electronics design and production is as follows:

i. preamplifier hybrids

The design of the custum monochip is complete and we have about 1500 chips in stock supplied by Argo Transdata. A total of $20 \mathrm{~K}$ monochips is needed and we ha: $A$ gone out for tender to several companies.

ii. calorimeter data units (CDU's)

The first 150 units have been ordered from Interconnect Technology. They now being tested and assembled into dual-CDU packages. A total of 2880 dual-CDU packages are needed.

iii. preamplifier motherboards

The first 10 motherboards have been ordered from Interconnect Technology. One motherboard is needed per position detector module for a total of 480 .

iv. analog-to-digital converters

The first prototype has been completed and debugged. A total of $36 \mathrm{ADC}$ bords are needed.

v. time sequencer

A prototype version has been completed and is undergoing design review. Only one sequencer is needed.

vi. memory address sequencer

A prototype version has been completed. Only one sequencer is needed. 


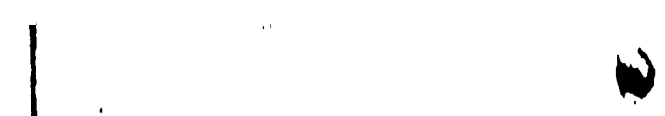

H)

vii. flex receiver

A prototype version has been completed and is being debugged. The design of the flex receiver is under review. 


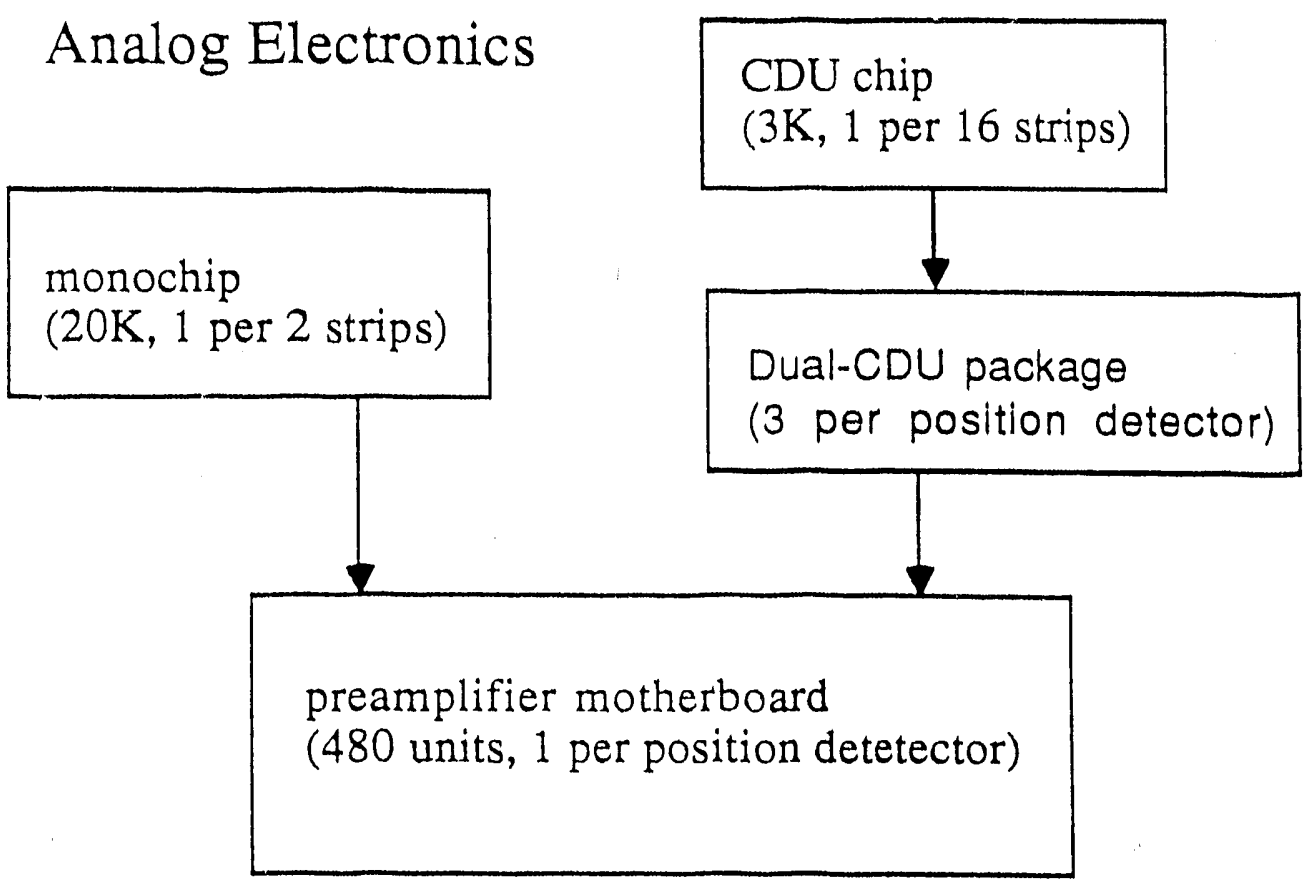

\section{Digital Electronics}

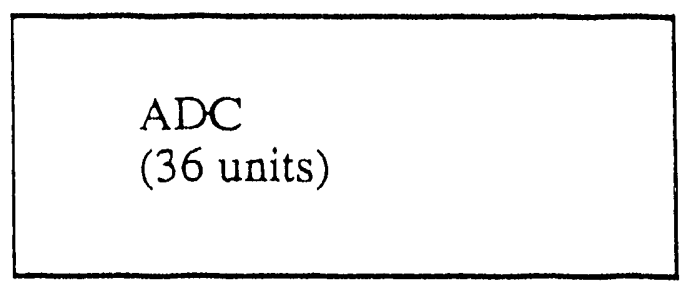

Time sequencer

(1 unit)

Memory Address Sequencer

(1 unit)

Flex Receiver (36 units)

Figure III.2 Main components of the position detector electronics, indicating quantities needed. 


\section{d) Results of Beam Tests}

Measurements were made in the SPS test beam at CERN during September 1987, and again in the PS test beam during November 1987. The position detector was mounted just upstream of the prototype uranium-TMP calorimeter. A target of 4 to 6 uraniurn plates of $2 \mathrm{~mm}$ thickness spaced by $3 \mathrm{~mm}$ was placed directly in front of the position detector. The incident angle of the beam was varied to simulate different parts of the barrel configuration of the final calorimeter. A multiwire proportional chamber was used to measure the position of the beam. Eight horizontal and 8 vertical strips of the position detector were connected to preamplifiers of the type designed for the DELPHI calorimeter, designed to have low noise at low detector capacitance. The signal from each preamplifier was then shaped using a UAl calorimeter shaper ( 2 microsec shaping time constant) and the peak signal recorded with a LRS 2249 ADC. Electron trigger data were taken at beam energies of ranging from 3 to $50 \mathrm{GeV}$ for a variety of detector voltages up to $2500 \mathrm{~V}$.

\section{i. TMP Lifetime}

Total charge was collected on a single strip (waveform data) averaged over many showers. The electrunics used after the preamplifier (test pulse, filter, and flash-ADC) was the same as that used for measurements on the prototype calorimeter. Waveform data were taken at several voltages between 4 and 2500 volts. Charge was digitized in $50 \mathrm{~ns}$ bins except for the lowest voltage data which were digitized every $100 \mathrm{~ns}$. The purpose of the low voltage measurements is to measure the TMP lifetime. The high voltage measurerments were taken in order to measure the drift time.

The most sensitive measurement of the TMP lifetime comes from the data taken at a voltage of 4.4 volts. This measurement was found to be reproducible. The result of this fit to the TMP lifetime is

$$
\tau=57.7+31.2-16.8 \mu \mathrm{s}
$$

The errors on the fit are asymmetric; the result is consistent with a nearly infinite lifetime on the high-end and is inconsistent with about $40 \mu$ s on the low-end. 

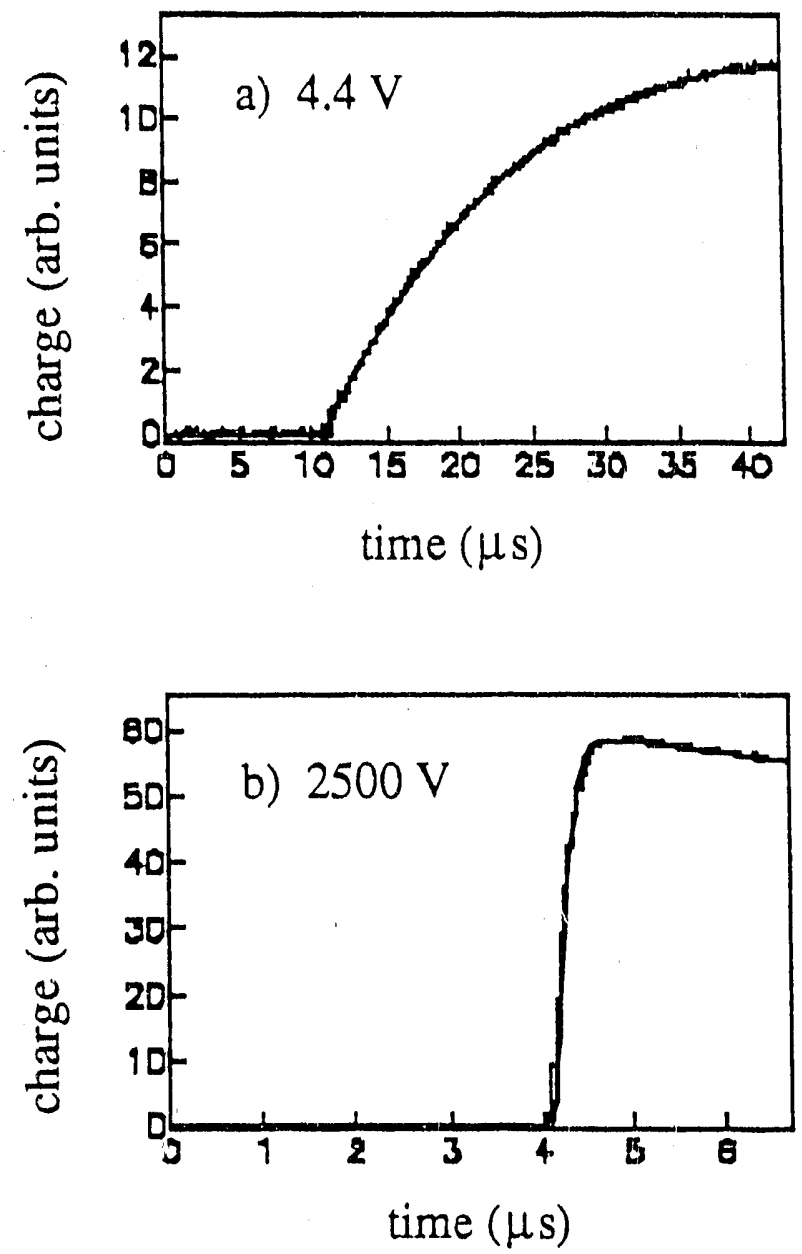

Figure III.3 Integral charge collected vs. time on a single strip of position detector summed over 1000 showers for a) very low voltage to measure charge attenuation and b) high voltage. 
ii. Drift Velocity

The electron drift time $\left(t_{d}\right)$ across a gap of distance $d$, is given by the relationship $t_{d}=$ $\mathrm{d}^{2} /(\mu \mathrm{V})$, where $\mu$ is the TMP mobility of $30 \mathrm{~cm}^{2} / \mathrm{V}$-s and $\mathrm{V}$ is the voltage across the gap. Figure III. 4 shows a plot of the measured inverse drift times $t_{d}^{-1}$ vs. voltage on a log-log plot. The inverse drift times are seen to increase linearly with voltage without saturation over the measured range, showing that the TMP mobility is a constant. At $2.5 \mathrm{KV}$ the charge is collected in about 200 $\mathrm{ns}$ in the $1.25 \mathrm{~mm}$ gap.

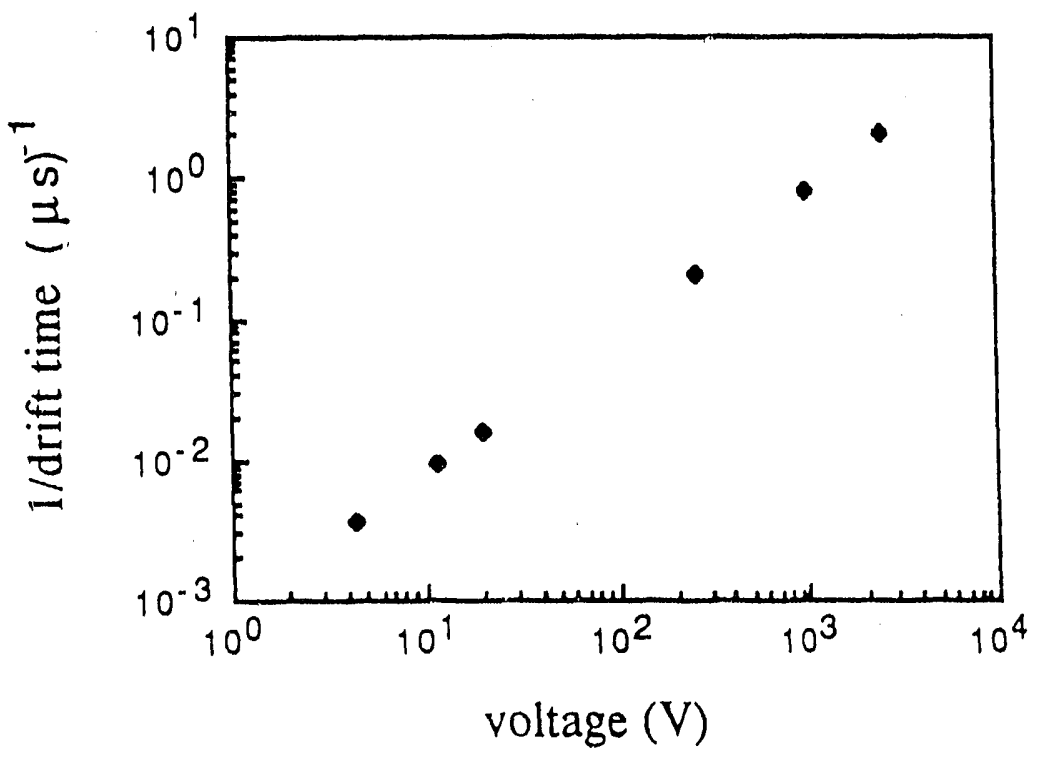

Figure II. 4 Measured inverse electron drift time across $1.25 \mathrm{~mm}$ gap vs. voltage applied across position detector gap. 
iii. Response to Minimum Ionizing Particles

The position detector response to minimum ionizing particles was measured with a muon beam. Figure III.5 shows the pedestal fluctuation together with the muon signal observed when the muon passes through the enter of a strip (within $\pm 2 \mathrm{~mm}$ ). The rms fluctuations in the noise are about 1500 electrons, and the mean value of the muon signal is about 3000 electrons.

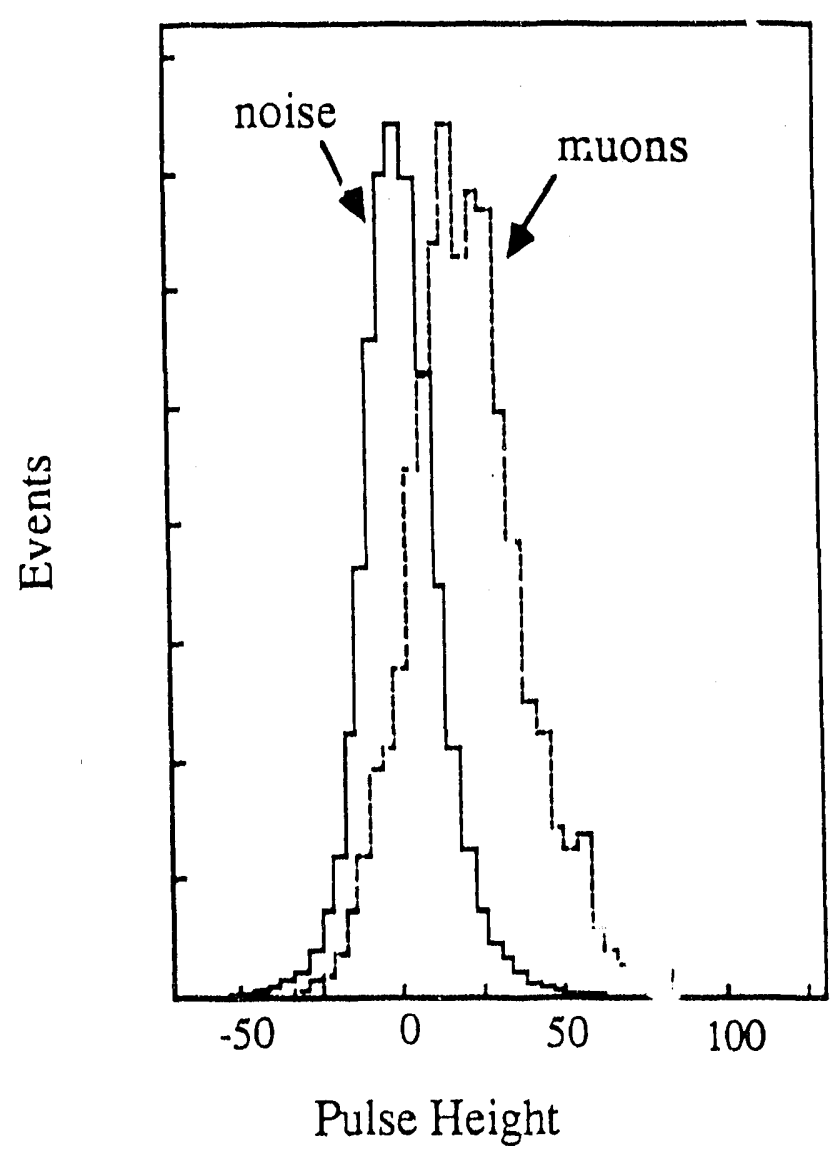

Figure III.5 Response of minimum ionizing muons on single strip of position detector compared with noise measurments on the same strip. 
iv. Response to Different Beam Energies

The total position detector signal was measured ( $x$ and $y$ strips separately) for a range of beam energies. The data taken at normal incidence with a target of 6 uranium plates is shown in fig. III.6.

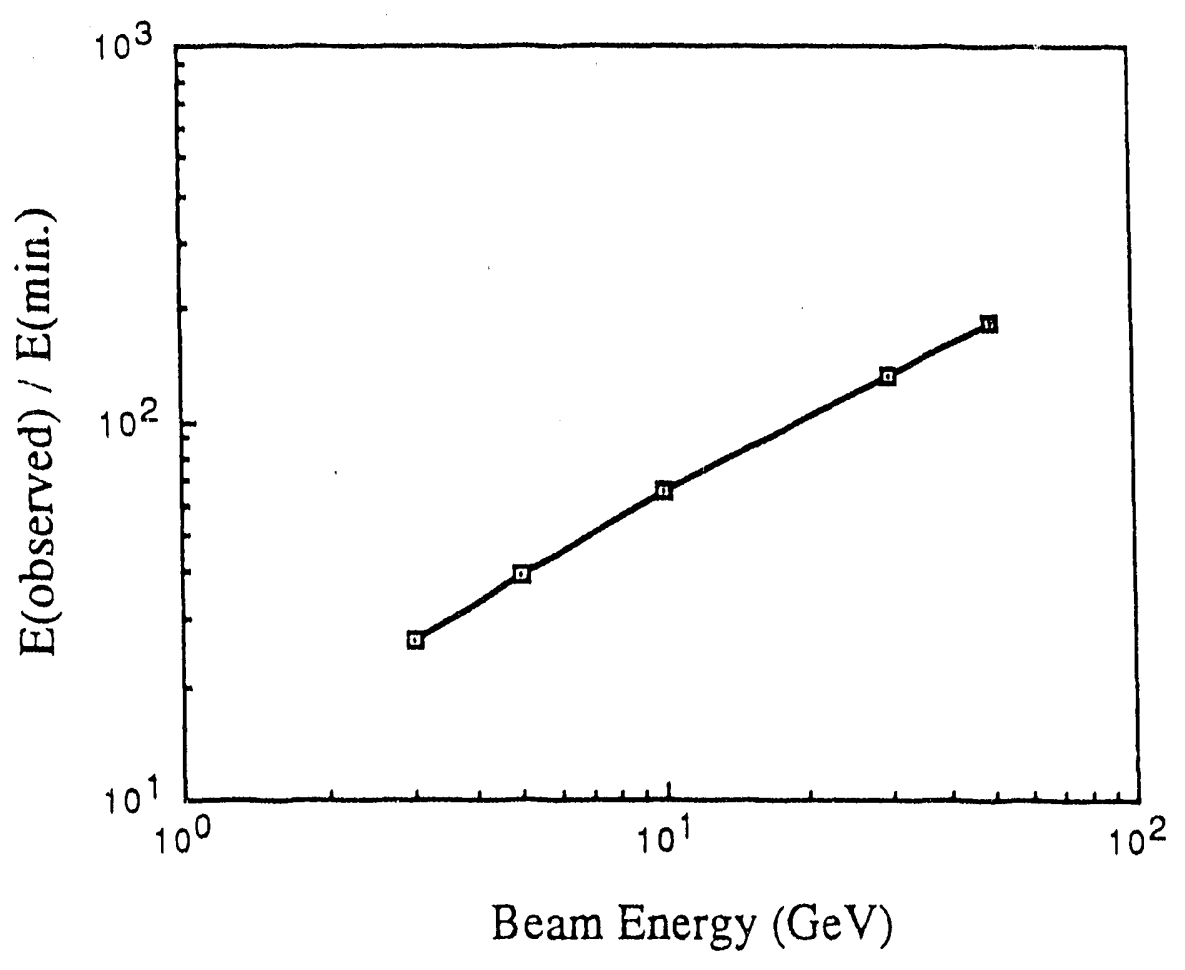

Figure III.6 Observed average signals, normalized to single minimum ionizing, in position detector for different energy positron showers. 
v. Strip Hit Distributions

Figure III.7 shows histograms of the average pulse height fraction seen on each strip (\% per strip) as a function of the beam position in $1 \mathrm{~mm}$ steps. The beam energy is $10 \mathrm{GeV}$.
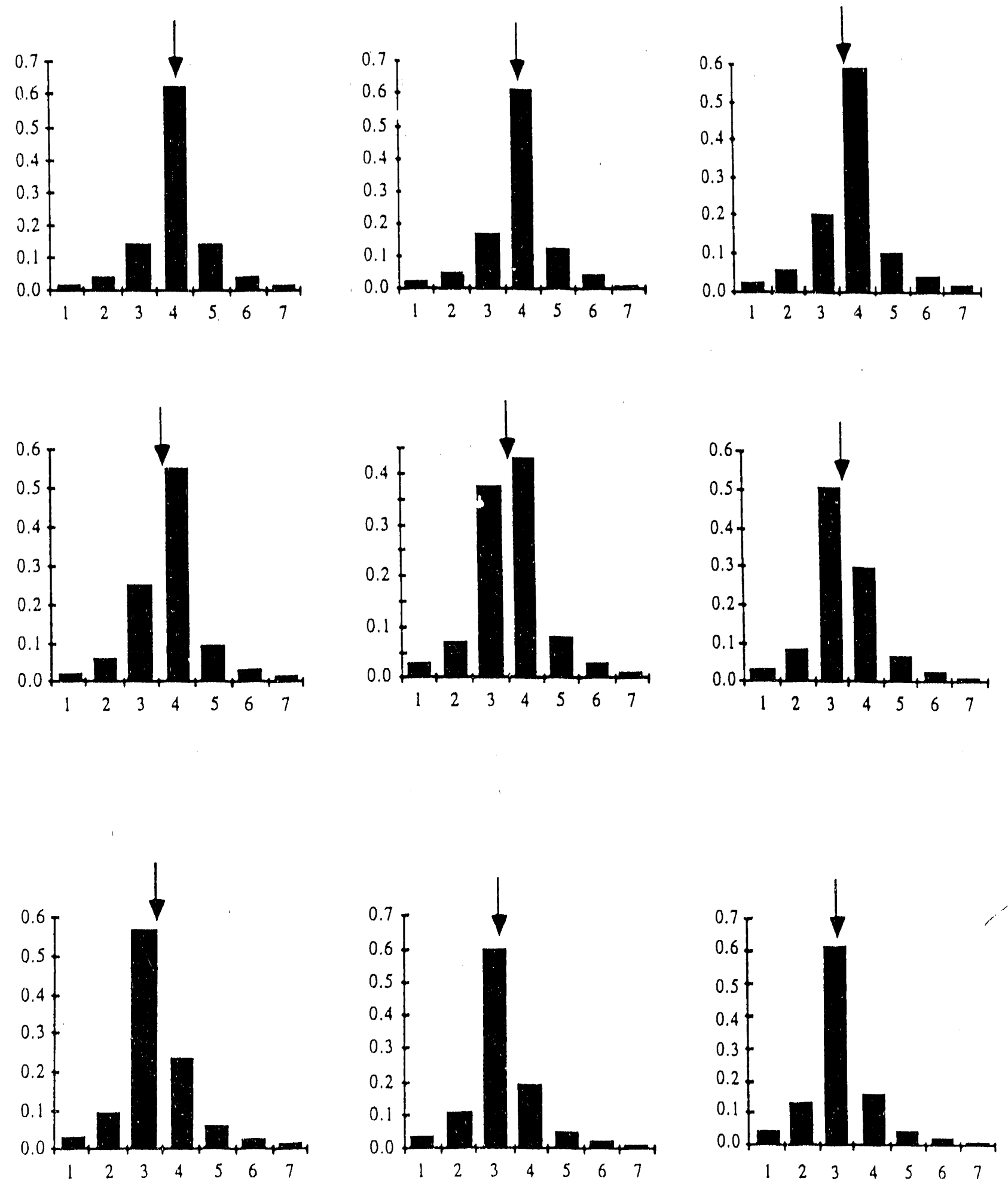

Figure III.7 Average energy measured on each strip for $1 \mathrm{~mm}$ steps in beam position (indicated by arrows). 
vi. Uniformity of Detector Response

Figure III.8 shows a plot of the total pulse height measured normalized to minimum ionizing summed over all y-strips vs. the beam position for a $10 \mathrm{GeV}$ beam. A uniform total response is observed independent of shower location.

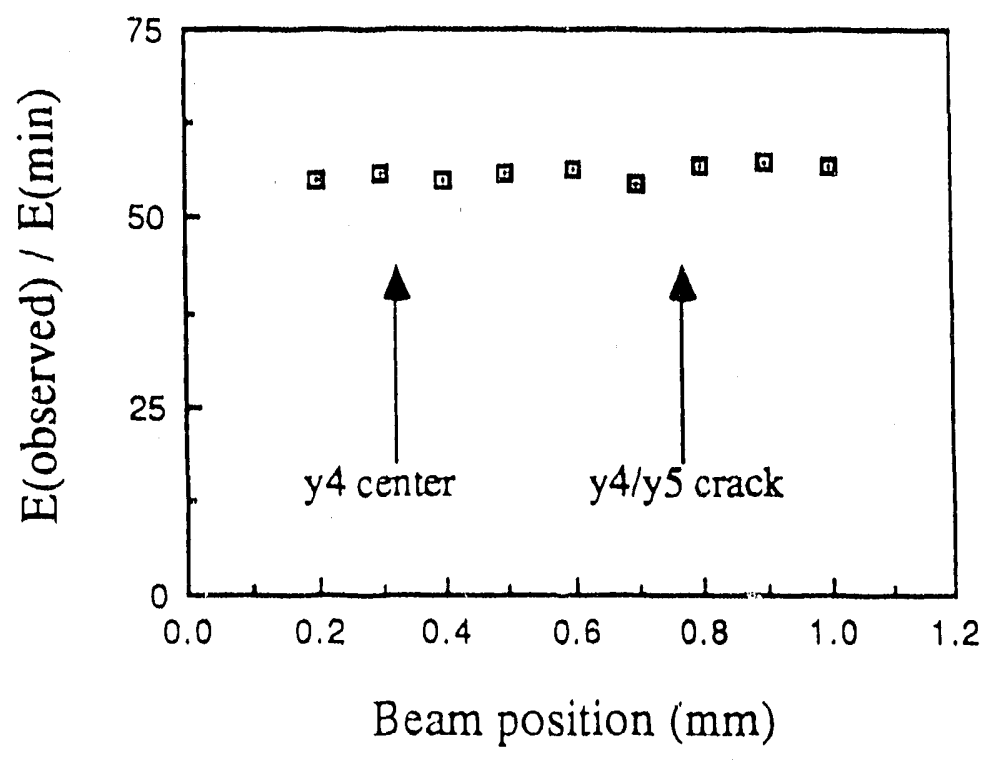

Figure III.8 Observed signal, normalized to minimum ionizing, as a function of shower position; the arrow on the left indicates the position of a strip center and the arrow on the right indicates the position of a crack between two strips. 
vii. Position Resolution

The position resolution is a function of the amount of energy deposited in the position detector which depends not only on the shower energy (beam energy) but also on the local shower fluctuations. Figure III.9 shows the resolution, calculated as full-width-half-maximum divided by 2.4 , using the simple centroid calculation. The resolution is plotted vs. observed signal (normalized to minimum ionizing) for various beam energies. Figure III.10 shows the resolution observed for the case of large pulse height deposited by a $50 \mathrm{GeV}$ shower.

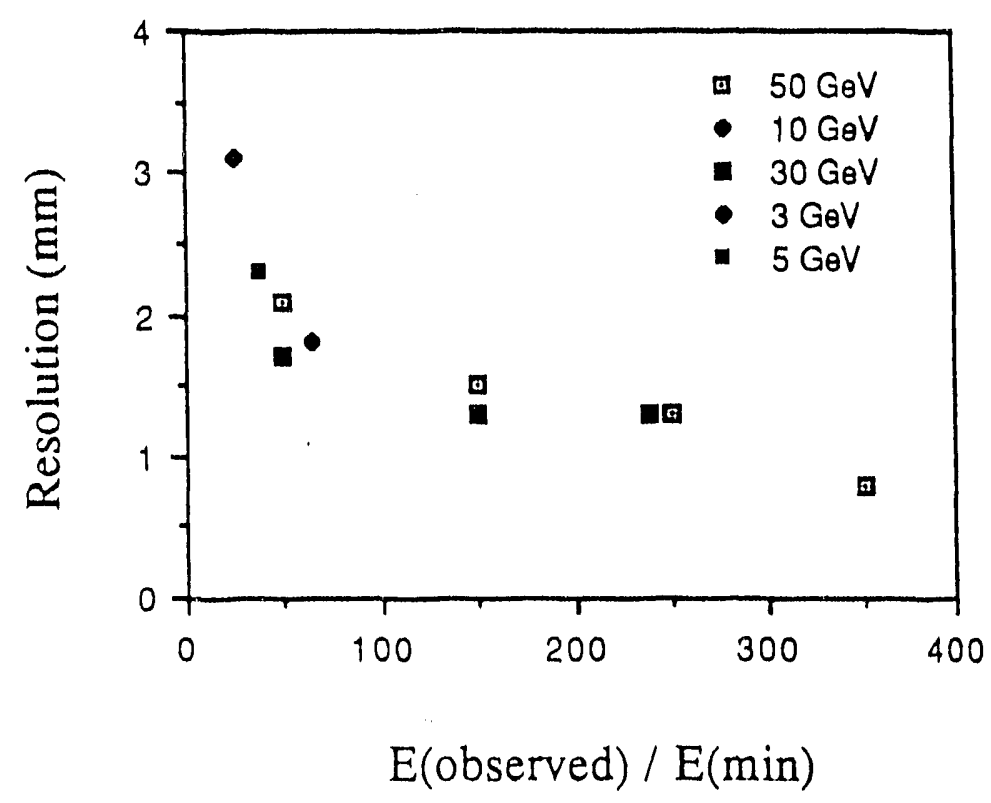

Figure III.9 Position detector resolution (mm) measured for various observed signals from different shower energies. 
a) x-strip residuals

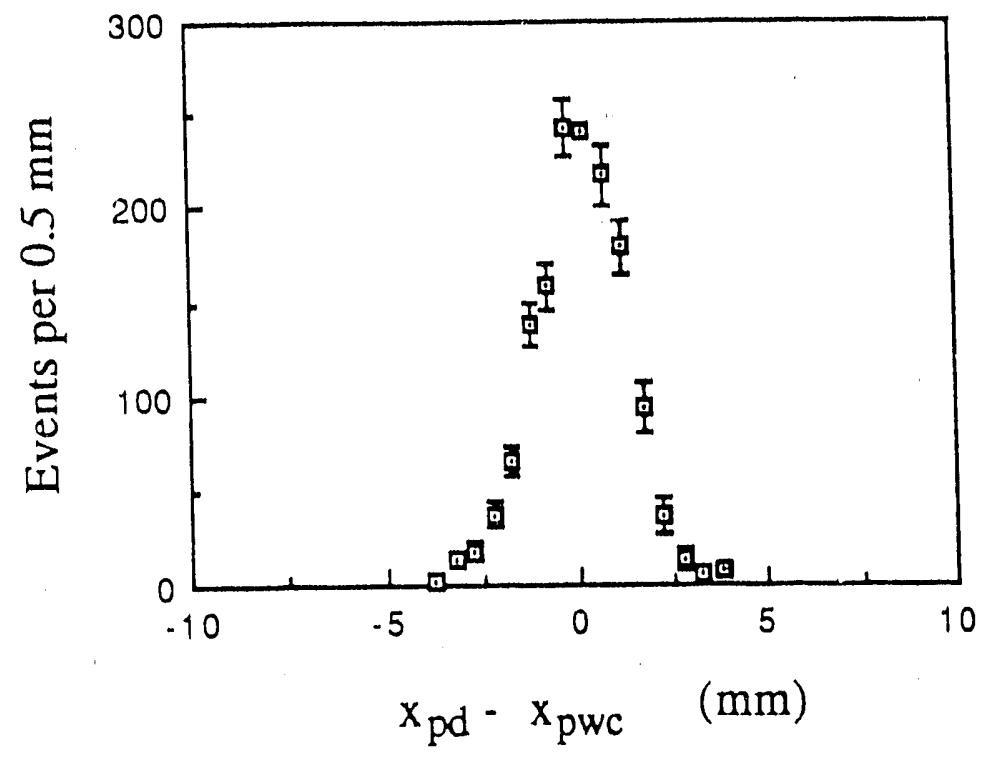

b) y-strip residuals

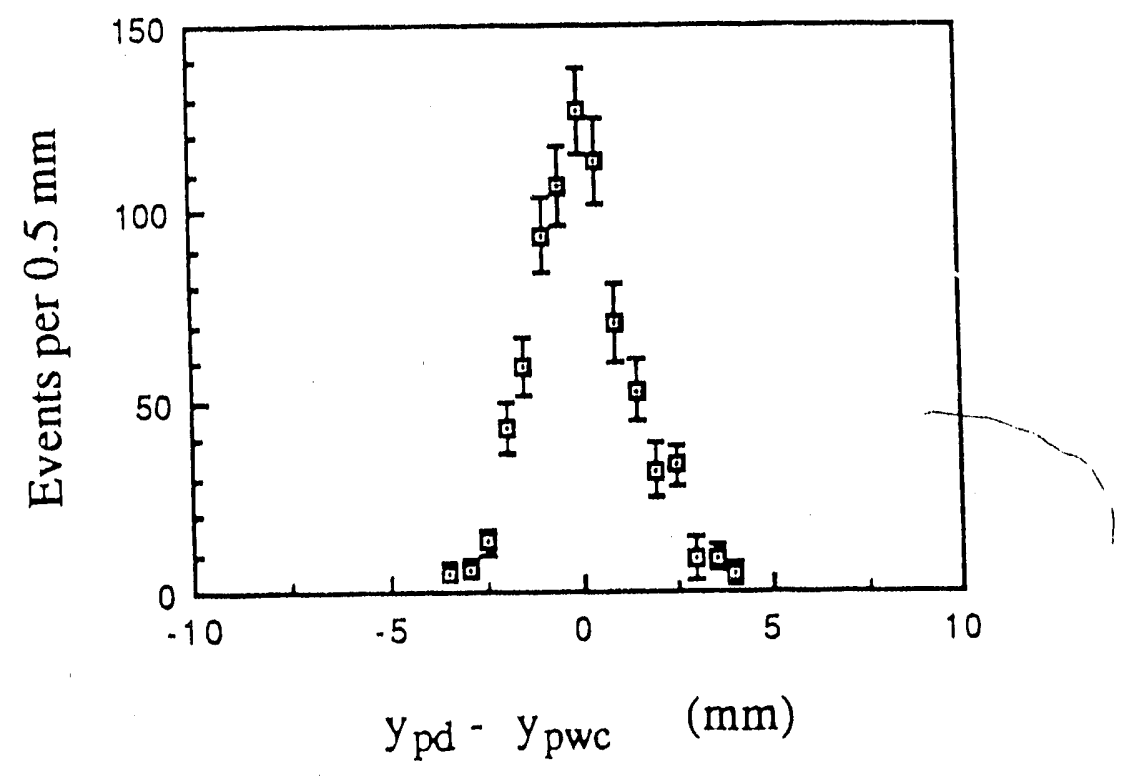

Figure III.10 Residual of the shower position for $50 \mathrm{GeV}$ showers as determined by center of gravity in the the position detector and the wire chamber for a) vertical strips and b) horizontal strips. 

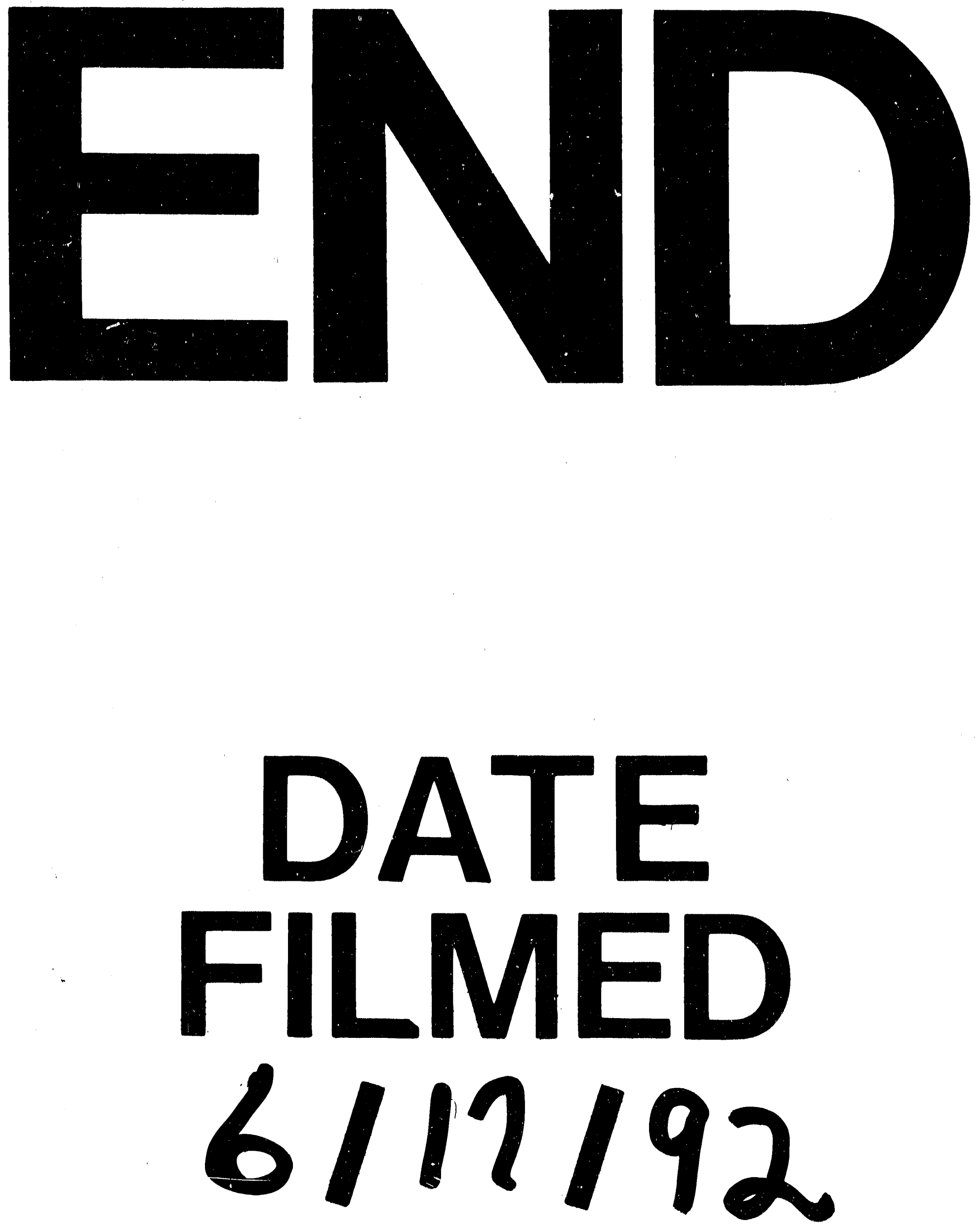
